Unlike hæmorrhage, perforation demands the permanent suspension of bathing, and there can be no doubt that Dr. Caiger is right in stating that laparotomy, and laparotomy at the earliest possible moment, is in almost all cases the only correct procedure. Writing in 1897 I said: ${ }^{5}$ "There can ...... be little doubt that the treatment of perforation, in hospitals at any rate, will in future be mainly by laparotomy; and that those hospitals in wbich the routine treatment is by systematic cold bathing will provide by far the largest supply of suitable cases for this operation." The reason for this is clear. "Systematic bathing ...... has so altered the symptoms of this accident and rendered so certain the possibility of its immediate diagnosis that the whole question of treatment requires reconsideration. Under expectant treatment the diagnosis is not infrequently impossible at the time. At the Brisbane Hospital up to the end of 1886 it was not uncommon to find perforative peritonitis for the first time in the post-mortem room. ...... In contrast to this is the experience of the Brisbane Hospital during the first three years of the bath treatment. During this time 34 cases suffered perforation, but so clear was the mental condition in all these patients that in every instance they were able to fix almost exactly the time of the accident. Not only so, but they were able as a rule to give so significant an account of the onset, site, and character of the pain, that usually a positive diagnosis could be made even before the signs of peritonitis were apparent."

The history of many cases was a sudden pain in some definite part of the abdomen; later an access of vomiting with a concurrent exaggeration of the pain, indicating extravasation of bowel contents. And it was found that very often the administration of a hypodermic injection of morphia (one-third to one-half grain) immediately after the commencement of the pain was capable of deferring the onset of vomiting for some hours. That is the opportunity for laparotomy in my opinion.

I am, Sirs, yours faithfully,

$$
\text { Francis Everard Hare, M.D. }
$$

\section{THE TREATMENT OF STRABISMUS.}

\section{To the Editors of THE LANCET.}

SrRs,-As the brief account of my operation for strabismus, which appeared in THE LANCET of Jan. 7th, p. 61, is not sufficiently clear to prevent the possibility of misinterpretation, I should like to supplement it by a few remarks of my own.

My principal object is to replace as much as possible tenotomy by the "advancement" of the muscles in the operation for all imperfections of the motility of the eyes (paralytic as well as concomitant squint, insufficiency, \&c.). During last year I performed 100 "advancements," always with very good results, but in the same period I performed only two tenotomies.

There are some rare cases of convergent strabismus of very old standing, with strong contracture (not contraction) and marked alteration of the structure of the internus muscle.
FIG. 1.

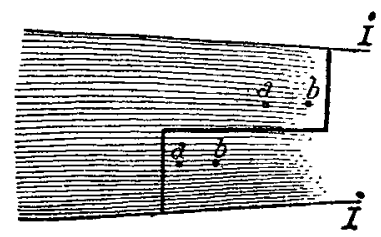

Lines of incision in muscle. $a$, $b$, Points of suture.
FIG. 2.

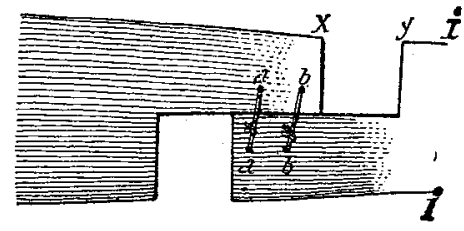

Muscle arvanced and sutured. $x, y$, Gap in muscle. The remaining letters signify the same as in Fig. 1
A surgical operation on this muscle seems to be admissible simultaneously with "advancement" of the opposing muscle. Only, instead of setting back the shortened internus by a tenotomy, I propose to preserve its normal insertion on the eyeball and to lengthen it by a special procedure. The new operation consists in severing the muscle so that the divided extremities are left somewhat in the form of steps of a stair as shown in Fig. 1, and lengthening it by uniting these

5 The Cold-bath Treatment of Typhoid Fever, F. E. Hare, 1898 pp. 186 et seq. extremities, as shown in Fig. 2. The operation has already given me excellent results.

Paris, Jan. 17th, 1905 I am, Sirs, yours faithfully,

\section{THE PRINCIPLES OF TREATMENT OF PNEUMONIA. \\ To the Editors of THE LANCET.}

SIRS, - In a rather remarkable address on the Principles of Treatment of Pneumonia, published in THE LANCET of Jan. 21st, p. 138, Dr. W. Ewart lucidly presents his views and his practice as derived from numerous and careful clinica observations and also from certain conceptions as to the pathological processes in the lungs and elsewhere. It is, of course, on this broad basis that all successful treatment must be reared and tested. Regarding the directing of treatment in conformity with what one conceives to be the pauhology of the disease, this, though a most laudable ambition, is sometimes hazardous, and should no ill-results follow, the treatment may be right; but, on the other hand, the patient may recover in spite of the treat ment. As to the indications for treatment which are supplied by the pathology of pneumonia I venture to notice briefly two of these, both of high importance, though differing widely in range. One of them is considerably exploited by Dr. Ewart; the other is not alluded to directly in his address but is suggested by his acute observations. That discussed by Dr. Ewart refers to the influence and import of the fibrinous coagulation in the lungs. $\mathrm{He}$ begins by quoting the opinion of a New York physician to the effect that "the extensive effusion of fibrin is really a ' breeding ground' for the diplococei and this enormous supply of pabulum is the explanation for the overwhelming amount of poison manufactured by them in the lung and thrown into the circulation." Then in discussing the principles of action, Dr. Ewart advocates "defibrination," observing that "to discourage the local consolidation by diminishing the supply of fibrin factors is the end towards which our treatment and diet are made to converge." Further on it is explained that the consolidation is checked by administering citric acid, a precipitant of calcium which is essential to clotting. Other measures to this end are also advocated, including the possibility of impairing the solidification of the fibrin by withholding salt from the diet in view of the fact that the fibrinous exudation is rich in sodium chloride.

In studying the pathology of this disease for several years nothing more strongly impressed itself on my mind than the view that the pneumonic consolidation is nature's method of localising the morbid process and of curing the disease. The statement quoted by Dr. Ewart as to the fibrinous exudate forming a breeding ground for the pneumococcus belongs to the realm of fancy and is neither scientifically correct nor borne out by actual observation. In the first place, the fibrinous exudate is dead, in a state of coagulation necrosis, and so does not offer a suitable pabulum for the micro-organism. The organisms, imprisoned in this rigid dead substance, behave as they do in certain artificial media-viz., secrete an acid which quickly inhibits their vitality and their virulence. Some have supposed that this acid in the pneumonic lung is secreted by the pulmonary tissues and have termed it pneumic acid, but when engaged a few years ago in a research on this subject I extracted from pneumonic lungs an acid giving identical reactions to that obtained in artificial cultures. In examining microscopically sections of pneumonic lung in various stages of hepatisation I have been struck with the absence of active growth of pneumococci within the alveoli. Moreover, in cases of pneumococcus infection showing little or no tendency to pulmonary hepatisation; when the pneumococci run riot in the organism so to speak, the result is always grave and, indeed, generally fatal. Consolidation is doubtless the natural and wholesome reaction of the tissues to the disease, and though any attempt to modify the virulence of the organism and in this way limit the extent of the consolidation is not only justifiable but the object most to be desired, yet any inter. ference directly, as by drugs, with the normal deposition of fibrin is, according to the view here propounded, simply to direct one's weapons against the vis medicatrix nature.

The other topic suggested by Dr. Ewart's address refers to the definitely scientific treatment of this affection in accordance with certain recent discoveries. Alluding to the objects 
of treatment, such as the arrest of the morbid process, the restriction of the invading host, and the neutralisation of the poison, Dr. Ewart remarks that the individual means to these ends may be inadequate at first or even mistaken. Inadequate doubtless, but perhaps not so much mistaken, provided our means are checked by the very definite method of blood examination which has been elaborated by Leishman and by Wright and Douglas. The amount of protective substances in the blood: those natural antitoxins which the latter observers have rather happily termed "opsonins," can be accurately estimated in any individual case of infectious disease and affords a true index of the patient's condition. The process is rendered both easy and speedy by the now celebrated method of Leishman, as modified by Wright. That the blood serum (not the corpuscular elements) contains a substance (an opsonin) which effects a chemical interaction with the bacteria so as to prepare the latter for phagocytosis seems to have been definitely determined quite recently by Wright and Donglas. In the case of two specimens of blood of conspicuously different phagocytic power the corpuscular elements were separated from the fluids and an interchange effected. "The leucocytes of the successfully immunised patient exhibited in these circumstances the lesser phagocytic action characteristic of the blood of the normal individual who served as a control, while the leucocytes of the normal individual exhibited the increased phagocytic action characteristic of the blood of the successfully immunised patient." By counting the number of organisms (from an artificial culture) which the washed cells of normal blood when mixed with the patient's serum can ingest under certain conditions an accurate estimation can be made of the opsonic content of the blood. This phagocytic count is compared with the phagocytic count of normal blood, taken as unity, ${ }^{2}$ and so from hour to hour or from day to day the rise or fall in the protective substances in the patient's blood can be accurately determined. Although at first sight it might be supposed that the results thus obtained would be capricious, yet repeated observations made in cases of acute and chronic infections show the method to be remarkably accurate. As already observed, this protective substance or opsonin is normally present in the blood, but when a vaccine is introduced into the patient's system its effect is to cause a greatly increased elaboration of opsonins by certain cells of the body. In the case of pneumonia we have no vaccine as yet which can be administered. But one can see that an attempt at a really scientific treatment of the disease would be made if the various methods of treatment adopted were considered in conjunction with the rise or fall of the opsonic curve. If, for instance, it was found that alkaline treatment was accompanied by a rise in the opsonic curve this would indicate that benefit was being derived, and vice versâ. In short, it might be possible by an organised series of observations to determine treatment generally applicable to the disease and also that adapted to its varieties and special phases. And it may be remarked that the clinical symptoms alone are not always a safe guide. The opsonic curve may be falling with but little change in the symptoms, for a time at least. In certain inoculations I made lately on animals I found in certain cases the opsonic value of the blood falling without any loss of body weight or other clinical symptoms to indicate it. At any rate we have here opened up the possibility of treatment based on scientific data of a very precise character. It does not include the entire treatment of the disease, more especially as to the mechanical aspects, but it covers a very wide field and may in time displace the theorising and groping which up to the present have more or less characterised the efforts of the physician.

I am, Sirs, yours faithfully,

Henrietta-street, W., Jan. 21st, 1905.

A. G. Auld.

\section{ANKYLOSTOMUM DUODENALE.}

To the Editors of THE LANCET.

SiRs,-Upon my return to Egypt I find that in a communication by Dr. Alessandrini to the Italian Zoological Society, written at the end of 1904, I am given the credit of having made independent research apon the question of the migration of the larvæ of the ankylostomum duodenale from

1 Proceedings of the Royal Society, Sept. 28th, 1904.

2 In each case one volume of serum is adder to one volume of corpuseles from citrated normal blood and one volume of a suspension of the organisms. the skin to the intestine. I also hear that other authors such as Bentley and Zinn, seem to have accorded me similar credit. I therefore ask for a few lines of your valuable space to state that my two communications on this subject were written by Professor Looss's authorisation as a means of informing English readers of his now well-known discoveries.

Cairo, Jan. 12th, 1905 I am, Sirs, yours faithfully

\section{FRACTURE OF THE ANTERIOR FOSSA OF THE SKULL DIVIDING THE OPTIC NERVE. \\ To the Editors of THE LANCET.}

SIRS,-In The LanceT of Jan. 21st Dr. F. J. Hathaway records a case of the above and it is commented upon in your annotations. Will you permit me to say that I discussed the same question in a paper before the Ophthalmological Society a few years since? I then recorded 18 cases and treated the subject somewhat fully in a paper which occupies 25 pages of the Transactions of the society for 1897. It is briefly abstracted in THE LANCET of June 26th, 1897, p. 1745. Since that time $I$ have seen fully a dozen additional instances. Three cases a short while ago were attending my clinic at the same time and were shown at a meeting of the Sheffield Medico-Chirurgical Society. One case is at present among my out-patients at the Sheffield Royal Infirmary. I am not therefore disposed to consider the association of damage of the optic nerve with head injuries as particularly rare and, if looked for, such cases in a large clinic will not infrequently, I believe, be met with.

The medico-legal aspect of these cases is interesting and important. An individual meets with an injury to the head; there may be some loss of consciousness or not and the general symptoms pointing to severe head injury may or may not be marked but he asserts that he has thereby completely lost the sight of one eye. Examination with the ophthalmoscope only yields negative results. The lesson to learn from these cases is, I think, that one should be guarded in forming an opinion and should recognise the possibility of the patient's statement being correct. This will be corroborated, if true, after a lapse of a few weeks (three or more) by the decolouration which will be noticeable in the optic papilla. I referred to this matter in the paper I have mentioned and it has also been my habit to point out to my class for some years its importance. The absence of ophthalmoscopic evidence at the outset is doubtless due to the injury being posterior to the point of entrance of the central retinal artery into the optic nerve.

Sheffield, Jan. 23rd, 1905 I am, Sirs, yours faithfully,

\section{HESSING'S SPLINT AND CONTRACTURE AT THE KNEE.}

To the Editors of THF LANCET.

SrRs, - I should be obliged if you would grant me space wherein to correct two omissions in my recent communication. First, the instrument I made use of was Hessing's splint-case appliance as modified by Hoefftcke. One of the modifications is a very important one and consists in an arrangement at the ankle whereby continuous traction of the leg is applied during the straightening of the knee. It is this traction which prevents the straightening process from causing pain. Secondly, I omitted to mention that the mobility of the knee, which at the commencement of treat. ment was almost imperceptible. has very slowly but steadily increased, being now some 15 degrees'; in other words a flexed, rigidly ankyłosed knee has been transformed into one with fair and apparently increasing mobility. I am, Sirs, yours faithfully,

Wimpole-street, W., Jan. 24th, 1905.

T. H. OPENSHAW.

\section{WEST AFRICA AS A HEALTH RESORT. To the Editors of THE LaNCET.}

SIRS,-I should be much obliged if you would be good enough to contradiet a report attributed to me that West Africa is likely to become a health resort. The health of Europeans in many of the coast towns now shows a marked improvement and will no doubt continue to improve. This 